\title{
\#BFAST INDEXING
}

bfast fasta2brg - $f$ vvinifera_all.fasta -A 1

$\mathrm{I}=0$

for $M$ in 111111111111111111111111111010011111001111111111110111111011001100011111000111111

1111111100101111000001100011111011111111110001111110011111111

111110110100110000110001100111111111111111111110011101111111111011000011111111001111011111

1110110001011010011100101111101111111111001000110001011100110001100011111

do

bfast index -n 20 -f vvinifera_all.fasta -A 1 -m \$M -w 14 -i \$l

$\mathrm{I}=\$((\mathrm{I}+1))$

done

\# READ PREPROCESSING

zcat sample.csfastq.gz | sed "s/^!//" > sample.bfast.csfastq

\# BFAST ALIGNMENT

bfast match -n 20 -f vvinifera_all.fasta -A 1 -r sample.bfast.csfastq -K 100 -M 500 > sample.bmf

bfast localalign -n 20 -f vvinifera_all.fasta -A 1 -m sample.bmf -o $10>$ sample.baf

bfast postprocess -n 20 -f vvinifera_all.fasta -A 1 -i sample.baf -a 3 > sample.sam

\# INDEL REALIGN

gatk -R vvinifera_all.fasta -I sample.bam -rf BadCigar --allow_potentially_misencoded_quality_scores -T LeftAlignIndels -o sample.re.bam

\section{\#VARIANT CALLING}

gatk -R vvinifera_all.fasta -I sample.re.bam -rf BadCigar --allow_potentially_misencoded_quality_scores -T UnifiedGenotyper -o sample.raw.vcf --genotype_likelihoods_model BOTH -nt 20 